\title{
Perceptions of HIV Testing Venues among men who have sex with men in London and the Midlands, UK
}

\author{
Rusi Jaspal \\ De Montfort University, Leicester, UK \\ Åbo Akademi University, Turku, Finland
}

\begin{abstract}
Undiagnosed HIV and late HIV diagnosis increase the risk of poor disease prognosis in infected individuals and of onward HIV transmission. It is vital to encourage regular HIV testing among men who have sex with men (MSM), a group disproportionately affected by HIV. A sample of 18 MSM from London and the East Midlands in England were interviewed regarding their perceptions of testing in the following three contexts - genitourinary medicine (GUM) clinics, community settings, and at home using a self-testing kit. The data were analyzed using qualitative content analysis. Perceived stigma from healthcare professionals, fear of being seen by significant others, and delays in being attended to were seen as barriers to testing in GUM clinics. While community settings were viewed as more accepting of sexual identity, concerns around homophobia and HIV stigma impeded access to testing for some individuals. HIV self-testing alleviated confidentiality concerns, but interviewees doubted the accuracy of the test results and worried about the lack of social support in the event of a reactive test result. Recommendations are offered for improving the acceptability of HIV testing in these contexts.
\end{abstract}

\section{Keywords}

HIV; HIV testing; HIV self-testing; men who have sex with men; sexual health 


\section{INTRODUCTION}

HIV remains a significant public health challenge. Although HIV is now considered a manageable chronic condition, it can lead to serious health problems and even death if left untreated. HIV surveillance data in the UK (Kirwan et al., 2016) indicate that $13 \%$ of people living with HIV are not aware of their positive serostatus, and that 39\% of the 6095 individuals diagnosed in 2015 received their diagnosis at an advanced stage of infection ${ }^{1}$. Late diagnosis can significantly undermine disease prognosis. Moreover, evidence demonstrates the all-round health benefits of early initiation of antiretroviral therapy (ART) regardless of CD4 cell count (The Insight START Study Group, 2015). ART also decreases the risk of onward HIV transmission, thereby functioning as a prevention method. However, HIV diagnosis and ART initiation are dependent on the individual's willingness to test for HIV.

In $2015,54 \%$ of new HIV diagnoses in the UK were among men who have sex with men (MSM) (Kirwan et al., 2016). It is recommended that sexually active MSM test for HIV every 3-12 months, depending upon their sexual behavior ${ }^{2}$. To promote regular HIV testing, services are now being expanded to non-clinical settings, such as LGBT community settings and gay saunas/ bathhouses, and to HIV self-testing kits. Although there has been research into potential barriers to HIV testing in MSM, there has been no recent study comparing MSM's acceptability of HIV testing in distinct venues. Accordingly, the present study focuses on MSM's perceptions of HIV testing in three important contexts, namely genito-urinary medicine (GUM) clinics, community settings, and at home using a self-testing kit.

\section{HIV surveillance in the UK}

HIV prevalence in the UK is approximately $0.16 \%$ of the population - Public Health England (Kirwan et al., 2016) estimates that approximately 101,200 people were living with HIV in 2015. According to the same report, approximately 47,000 MSM were living with HIV in 2015, and in London it was estimated that 1 in 7 was HIV-positive. For those who test positive, HIV testing is often the first step to engagement in HIV care (Gray et al., 2013). Yet, a recent study (McDaid et al., 2016) estimated that only 54.9\% of MSM test annually and that only $26.7 \%$ of MSM reporting high-risk sexual behavior had taken 4 HIV tests over the last 2 years. These data highlight a need to promote regular HIV testing among MSM.

The majority of GUM clinics in the UK provide an 'opt out' HIV testing service for

\footnotetext{
${ }^{1} \mathrm{~A}$ normal CD4 cell count range is $500-1600$ cells $/ \mathrm{mm}^{3}$. Patients diagnosed when their CD4 count has fallen $<350$ cells $/ \mathrm{mm}^{3}$ are considered to have been diagnosed at a late stage of infection.

${ }^{2}$ HIV: Testing, Public Health England https://www.gov.uk/guidance/hiv-testing Accessed 23

December 2017
} 
patients. In most clinics, a $4^{\text {th }}$ generation combined antigen/ antibody finger-prick HIV test is offered and usually administered by a health advisor. Additionally, an intravenous blood sample is screened for HIV and results are usually available within 48 hours. Increasingly, rapid finger-prick HIV tests are being offered in community settings, such as LGBT charities, sexual health charities, LGBT nightclub venues, and gay saunas. Many MSM reportedly prefer to test in these contexts (Thornton et al., 2012). In addition, HIV self-testing has been legal in the UK since 2014. Self-testing kits can be purchased online and, at the time of writing, cost 29.95 UK Pounds (approximately 40 US Dollars). The kit consists of a $2^{\text {nd }}$ generation antibody rapid finger-prick HIV test. The individual personally conducts the test and receives the results within 15 minutes. No interaction with a healthcare professional is required. Given there are now a range of contexts in which one can test for HIV, it is crucial to understand attitudes towards them.

\section{Barriers to HIV testing among MSM}

In previous research (e.g. Evangeli, Pady \& Wroe, 2016), several social and psychological barriers to regular HIV testing have been identified.

- Fear of mortality and illness as a result of HIV can lead some individuals to prefer not to know their HIV status (Lorenc et al., 2011).

- Some MSM do not view themselves as being at risk of HIV due, for instance, to low levels of awareness regarding HIV risk factors or because they do not self-identify as gay (Bond et al., 2015). People who perceive themselves to be at low risk of HIV are in turn less likely to test for HIV (Marcus, Gassowski \& Drewes, 2016).

- Endorsement of HIV-related stigma is negatively correlated with HIV testing in MSM (Li et al., 2012). Individuals may refrain from testing for HIV in order to avoid selfassociation with this stigmatized condition (Young, Nussbaum \& Monin, 2007).

- In the case of ethnic minority MSM, some forms of prejudice such as homophobia and racism are associated with decreased willingness to test for HIV (Bond et al., 2015).

Some of these barriers may be accentuated in some testing venues and attenuated in others. For instance, some MSM may anticipate homophobia from healthcare professionals and, thus, avoid testing for HIV in GUM clinics, while others may avoid testing in LGBT community settings due to fears of involuntary disclosure of their HIV status (Stutterheim et al., 2014; Thornton et al., 2012). 


\section{Testing in GUM}

HIV testing in GUM clinics can provide the opportunity for education and facilitation of behavior change to reduce one's risk of acquiring HIV or of transmitting it to others (e.g. Goodall \& Leen, 2011; Kall et al., 2012). On the whole, MSM in the UK express satisfaction with GUM services and many regularly test for HIV in this context (e.g. Kurka, Soni \& Richardson, 2015).

Some MSM express ambivalence about testing in GUM due to concerns around stigma, loss of confidentiality, and low trust in healthcare professionals (St Lawrence et al., 2015). Moreover, one's experience and satisfaction with GUM services is likely to underpin future willingness to test in this context (Nuwaha et al., 2002). Perceived discrimination and lack of empathy in healthcare settings can decrease willingness to test (Heijnders \& van der Meij, 2006). Accordingly, interventions have been developed to improve empathy in healthcare professionals (Edwards et al., 2006). Self-stigma can derive from perceived stigma in healthcare settings and may adversely affect engagement with health services (Rüsch et al., 2009), as well as HIV testing (Wong, 2013).

\section{Testing in community settings}

Mackellar et al.'s (2005) study of undiagnosed HIV and sexual risk behaviors among young MSM concludes with the recommendation that HIV testing should be expanded to gay bars, clubs and other social venues. The authors argue that the inclusion of testing in gay bathhouses and sex-on-premises venues would be effective in reaching MSM unaware of their positive serostatus (see also Woods et al., 2000; Bingham et al., 2003; Jaspal, 2018).

Rapid HIV testing in STI clinic studies and in bathhouse-based interventions have high acceptability among MSM (Kendrick et al., 2005; Spielberg et al., 2003). Prost et al. (2007) conducted a study of MSM's perceptions of rapid HIV testing in social venues as a means of exploring the provision of testing in non-clinical settings. They found that MSM might refrain from testing in these settings due to (1) concerns about lack of privacy, (2) the perception that social venues are inappropriate spaces for learning one's HIV status, (3) concerns about a possible lack of post-test support and about the types of behavior in which one might engage, and (4) fears that the provision of HIV testing could have an adverse effect on the venues in which it is offered. Furthermore, internalized homophobia can result in avoidance of HIV testing in LGBT community settings (Newcomb \& Mustanski, 2010). 


\section{HIV self-testing}

HIV self-testing can obviate many of the obstacles that individuals perceive in relation to HIV testing in GUM and community settings, and acceptability of HIV self-testing appears to be high among MSM (Figueroa et al., 2015). Convenience and confidentiality can facilitate HIV self-testing, while concerns around domestic confidentiality may impede it (Witzel et al., 2016b). Moreover, some people have experienced difficulties in utilizing self-testing kits (Johnson et al., 2014). There is dearth of UK-based research into attitudes towards HIV selftesting vis-à-vis other testing venues.

\section{METHOD}

\section{Participants}

Using a convenience sampling strategy, 18 HIV-negative MSM were recruited at two sexual health charities located in London and in the East Midlands of England. Participants were recruited at sexual health charities because the focus of the study was on sexual health and it was anticipated that participants recruited in these contexts would be relatively engaged around sexual health issues. Given the principal aim to obtain in-depth insight into participants' attitudes, a sample size of 18 was deemed appropriate. This is consistent with many other qualitative studies using qualitative thematic/ content analysis (e.g. Jaspal, 2018; Jaspal \& Daramilas, 2016). Participated were originally invited to participate in a social psychological study of sexual identity, health and behavior. Eligibility criteria for the original study included: being MSM, sexually active, and aged between 18 and 35 . The study received ethics approval from the Faculty of Health and Life Sciences Research Ethics Committee, De Montfort University Leicester, UK. Each participant read a detailed participant information sheet and signed a consent form prior to the interview. Each participated was reimbursed for their time with a $f 20$ gift voucher. HIV testing was a key focus of that study and constituted an important topic of discussion in the interviews.

Ten participants were recruited in London and 8 in the East Midlands. Fifteen individuals were White British and 3 were of South Asian heritage. Participants were aged between 22 and 32 years $(M=26.6)$. Twelve participants had university-level qualifications, and the remaining 6 had completed college education. All of the interviewees had reportedly tested for HIV in the past - 10 reported testing for HIV every 6 months, 5 reported testing once a year and 3 had had their last test over 1 year ago. Eight reported testing mainly in GUM clinics, 8 mainly in community settings, and 2 had used a self-testing 
kit. All participants had been tested in a GUM clinic in the past, which enabled them to compare this with testing in other contexts.

\section{Analytic approach}

The current article is a re-analysis of an existing data set focusing on the inter-relations between sexual identity, health and behavior. The distinct contexts for HIV testing emerged as an important topic of discussions and, thus, it was thought that this merited coverage in a separate article. Interviews were guided by a semi-structured interview schedule consisting of a series of exploratory, open-ended questions. The schedule included questions/ probes that elicited information concerning attitudes and experiences in relation to HIV testing in distinct contexts. Interviews were conducted by the author at De Montfort University, at a local sexual health charity, or over Skype. Interviews lasted between 60 and 90 minutes, and were digitally recorded and transcribed verbatim.

The data were analysed using qualitative content analysis, which enables the analyst to identify, analyse and describe patterns emerging in qualitative data (Hsieh-Fang \& Shannon, 2005). It is a flexible qualitative technique that allows the analyst to identify key perceptions of, and meanings attributed to, a particular phenomenon. This approach is used to shed light on attitudes and experiences in relation to HIV testing in distinct settings. There is a focus on how interviewees conceptualized their testing experience, the meanings they attributed to it, and how HIV testing might fit within broader perceptions of health and wellbeing.

The author transcribed the recordings and studied the transcripts. During each reading of the transcripts, preliminary interpretations were noted in the left margin. Then the right margin was used to collate these initial codes into overarching analytic observations, which captured the essential qualities of participants' accounts. These analytic observations are reported under the three subsections in the analysis section below. The interview extracts presented below are illustrative of the analytic observations.

\section{ANALYSIS}

The present section provides an overview of MSM's perceptions of HIV testing in three distinct contexts, focusing on the following themes: (1) GUM clinics: stigma and anxiety, (2) Community settings: a shared sexual identity, and (3) Self-testing: confidentiality amid decreased social support. In each subsection, participants' perceived advantages and disadvantages of testing in each of these contexts are discussed. 


\section{GUM clinics: stigma and anxiety}

All of the interviewees reported having used their local GUM clinic for regular HIV testing in the past. The majority of interviewees indicated overall dissatisfaction with testing in GUM and, thus, the focus of the interviews was largely on the disadvantages. An advantage for some participants was the availability of information and support from qualified healthcare professionals. Yet, primary concerns included the 'clinical atmosphere' at GUM clinics, which some individuals found unsettling:

I just start to feel really anxious, basically thinking 'am I sick?' I think it's because it's like a hospital in there. The same kind of smell and surroundings. It makes me feel really anxious. (Mark)

It makes me feel uncomfortable because it's all so 'hospital-y' and I'm not ill, am I? (Adam)

Taking an HIV test can be an anxiety-inducing event (Lorenc et al., 2011). Some individuals reported that the hospital-like environment of the GUM clinic further accentuated feelings of anxiety associated with testing because it evoked cognitions of ill health. Interviewees went for an HIV test hopeful for a negative result and, thus, did not perceive themselves as being unwell. Attendance at a hospital clinic therefore felt inconsistent with their identity. The clinical atmosphere of GUM clinics evoked an 'illness identity' which interviewees appeared to fear and, thus, wished to distance from their sense of self. This was construed as being inconsistent with their desired self, namely wellness. It is noteworthy that even those individuals who expected a positive HIV test result construed a positive serostatus as an undesired, feared event.

Amid feelings of anxiety that frequently accompany HIV testing, interviewees lamented the long queues that they habitually encountered in GUM clinics and the timeconsuming nature of HIV testing in this context:

I basically end up sitting there all day. I go there in the morning and sometimes I'm out just after noon, that's ridiculous, isn't it? It's almost like they're trying to discourage us from getting tested. (James) 
You're queuing and you're being looked at and I don't know what folks are thinking. Maybe 'oh he's got a sexual disease' and it makes me feel ashamed of myself a bit. (Peter)

In addition to the practical problem of accommodating sexual health screening in their routine, some respondents including Peter outlined their fear of stigma from others whom they might encounter as a result of the length of time they had to spend waiting to be seen. The long queues could add to the anxiety not only of being tested for HIV, but also of being seen by somebody who might make assumptions about one's sexual health status. As exemplified by Peter's account, feared or perceived stigma could result in negative emotional experiences, such as shame.

Some participants reported being denied access to sexual health services due to their sexual orientation, which could result in negative implications for self-esteem:

I was told to fill out a form and I put down that I have sex with men and the receptionist was like "we can't see you today because there is no doctor available" and I'm thinking "I don't need a doctor - I just want to get tested" (John)

Basically it's like if you're gay and you say it, it's a whole load of shit you've got to fill out and sort out. It's as if you're a problem patient which isn't a nice feeling. (Simon)

Being turned away from GUM clinics without clear explanations made some individuals feel that they were being discriminated against on the basis of their sexual orientation, as exemplified by John's account. They did not understand why being MSM should impede access to an HIV test in the absence of a doctor at the GUM clinic. Participants attributed this policy to social stigma. As Brian indicated in his account, this could induce a perception of negative distinctiveness on the basis of his sexual orientation, constructing him as a 'problem patient'. Moreover, some deduced from this policy that, as gay men, they were necessarily perceived as 'high risk', which has been found to constitute a possible barrier to accessing HIV prevention (Jaspal \& Daramilas, 2016):

Why am I 'high risk' just because I happen to like guys. Well, no, actually I'm not high risk because I always use condoms actually so that's not me actually. (Nick) 
The category 'high risk' can be threatening for self-esteem because of the social stigma appended to it. Stigmatizing attributes may be damaging for one's self-esteem and may consequently be distanced from the self-concept. Moreover, given the perceived mismatch between self-construal as 'safe' and others' categorization as 'high risk', some individuals like Nick experienced challenges to their sense of identity coherence.

Several interviewees anticipated 'intrusive' questions from receptionists and clinical staff, which also discouraged them from seeking an HIV test in GUM settings:

Joe: When the receptionist tells me to fill out the form I can sort of see it in her face, you know, that disapproval and then you get to the nurses and then they get started on all their questions and it's like one big nightmare.

Interviewer: Why?

Joe: They're sort of judging you even if they don't say it. Like saying 'well, if you stick to one guy, you won't be having any problems, will you?'

Like Joe, several interviewees perceived stigma from health professionals when they disclosed aspects of their sexual history, and remarked that health professionals lacked an understanding of gay men's lifestyle and sexuality. Even those stigmatizing reactions that are not overt can have an adverse psychological impact on some MSM. Some men reported disengaging from sexual health services apparently to protect their sense of self-esteem, which can be jeopardized by social stigma.

Others perceived judgment from clinical staff, which led them to provide inaccurate responses to questions. In some cases, this meant that they were not offered an HIV test:

At one point it got to a point where I was basically lying about being gay as well. I just said I'm straight and I really wanted an HIV test but they said I was low risk... I had done bareback a few times. (Bob)

Stigma can arise from the negative stereotypes associated with gay sexuality, namely that MSM invariably have multiple sexual partners and that they are unable or unwilling to sustain long-term monogamous relationships. Self-esteem was clearly compromised among 
some participants, and one strategy for averting threats to self-esteem was simply to deny, publicly at least, their sexual behavior and identity. This could give rise to erroneous risk assessment among health professionals, which in turn inhibited patients' access to HIV testing. For some individuals, this meant that they were not tested regularly or at all, while others actively sought an HIV test in community settings.

\section{Community settings: a shared sexual identity}

Almost half of interviewees felt more comfortable testing for HIV in community settings, whereby they anticipated less intrusive questioning and a quicker service. Accordingly, some preferred to test in these contexts:

What I've really appreciated about it [testing in an LGBT organization] is the fact that you go in there and they'll do it for you straight away. They cut to the chase really. You ask for it and you get it and that's all. It's what's needed. Not all the endless questions. (Mike)

For someone like me and I was thinking there's a high chance I've got HIV it is excruciating waiting to be seen and getting tested and everything. I just find that the waiting makes the whole thing more unbearable. HIV tests are never fun exactly, are they?... But when I go to [an LGBT organization] I get tested within minutes and get my result literally within minutes (James)

Several interviewees feared that, after a series of questions related to their sexual behavior, they might ultimately be refused a test in GUM settings. Conversely, there was a perception that in community settings it was possible to obtain an HIV test without having to persuade the tester of one's engagement in 'high-risk' behaviors and, thus, eligibility. Furthermore, as James indicated, the excessive waiting time in GUM clinics could cause further distress amid existing anxiety surrounding possible HIV infection. Some individuals perceived the questions that they faced in GUM clinics as unnecessary and instead wished for quicker access to testing. The rapidness of the testing process in community settings was advantageous.

Interviewees generally found the pre-test risk assessment to be less intrusive in community settings than in GUM clinics. Some derived comfort from the fact that, in most cases, HIV testers were gay themselves and were more understanding, and less judgmental, 
about their lives. This rendered the experience of testing for HIV in this setting more attractive:

When I go to [names an LGBT organization] I see the same tester and he knows me and he doesn't go into the whole detailed interrogation but yeah he asks me a few questions just to assess the risk really and then he does it. Because I know him and he's gay himself it's always a lot more comfortable. (Pete)

I'm not going to lie. I have a lifestyle that would raise eyebrows but the guy who I see when I go in [for an HIV test] is gay and it's nothing he hasn't heard before. (Jack)

Some participants reported having established a sense of rapport with their regular HIV tester with whom they felt comfortable discussing aspects of their sexual history. Jack recognized the stigma appended to his lifestyle in heteronormative contexts, which he contrasted with the acceptance and inclusion that he encountered in community settings. He reported feeling 'understood' and 'accepted' because the HIV testers manifested nonjudgmental and accepting attitudes towards his sexual identity. There was a desire among interviewees for greater understanding, acceptance and validation of their gay identities in testing contexts. Pete described the sexual health probing in GUM clinical settings negatively as a 'detailed interrogation', which he contrasted with the community setting HIV tester's 'few questions just to assess the risk'. Essentially, this enabled individuals to feel more comfortable about having an HIV test.

Given that most individuals positively evaluated their experience of testing for HIV in community settings, they reported doing so frequently, even when visiting community settings for other reasons. In short, HIV testing came to constitute a routine practice:

When I go in [to a gay sauna] for something else, sometimes you just kind of think 'yeah, why not take a test while I'm here?' because they're there doing their thing so you come that day and it becomes something of a routine really. (Stan)

Since there's the testing in the sauna I don't mind really about just doing it here. There isn't that massive worry or fear in a way. Stupid, I know, because it could come back positive but it just feels doable now. (Ken) 
It's the support and lack of judgment I suppose that makes it OK there [in the sauna]. (Joe)

Both Stan and Ken described the ease with which they tested for HIV in community settings - in their case, gay saunas. The availability of HIV testing in this context appeared to result in more frequent testing because individuals came to associate HIV testing with regular visits to the gay sauna. Ken attributed his ability to test for HIV to its availability in the gay saunas which he frequented on a regular basis. Moreover, Joe attributed his increased willingness to test in community settings to the 'support and lack of judgment' that he anticipated in that context.

Although HIV testing in community settings was generally evaluated in positive terms, interviewees did highlight possible barriers to testing in these contexts. For instance, some expressed concerns about being seen entering LGBT community settings due to the social stigma associated with homosexuality:

I don't want to be seen going in there because of the homophobia thing. You do still get quite a bit of homophobic people around and so I wouldn't want it to be common knowledge. I probably wouldn't do it. (Joe)

I walk away from the sauna sometimes because I'd die if I got seen going in basically and someone realizes I'm gay so yeah if I'm also there to get a test I'll just delay that too. (Mike)

For Joe and other participants, fear of homophobic discrimination induced trepidation about testing in these contexts. Some individuals feared negative social repercussions, such as being seen by a family member or a work colleague. It is noteworthy that interviewees differed in their level of 'outness' - not all of them were open to everyone about their sexual identities. Perceived homophobia can impede access to sexual health services offered in contexts perceived to be associated with LGBT identities. As Mike indicated, his desire to conceal his sexual orientation (due in part to perceived homophobia) also led to decreased access to HIV testing.

Some respondents feared that their use of sexual health services in LGBT settings could create the impression that they had engaged in sexual risk-taking behaviors. Participants' accounts highlighted not only the psychological significance of social stigma in 
inhibiting access to sexual health services but also the feelings of self-consciousness that could result from use of these services:

I mean the problem is basically that you go in [to an LGBT organization] and you don't know if you've been seen going in and they might be assuming that you either need help because you've got AIDS or something, and I don't want people thinking about me like that. (Nigel)

Everyone would just assume l've been sleeping around or done something or been stupid and got fucked bare or whatever because there's a lot of judging and that on the scene (Matt)

Sometimes I don't go [to test in a community setting]... I just don't want to admit it to myself, to be fair, you know that I've been a massive slut myself (Peter)

Interviewees expressed concern about social stigmatization on the basis of both their sexual orientation and assumed HIV status. Nigel was hesitant about visiting his local LGBT sexual health charity as others might assume that he had 'AIDS or something like that'. This evidenced participants' fear of HIV stigma. Peter did not wish to acknowledge his sexual risktaking behavior, but HIV testing in community contexts compelled him to do so and, thus, potentially to take action to reduce his risk.

\section{Self-testing: confidentiality amid decreased social support}

In contrast to HIV testing in GUM clinics and in community settings, HIV self-testing at home was perceived to be a 'safer', more confidential means of ascertaining one's HIV status. Several interviewees regarded this as more 'discreet' than other ways of testing:

I like the fact that you can just order it in and do it at home and nobody at all knows about it. When you go to the clinic or wherever, you're still interacting, isn't it? I don't like telling anyone I'm taking a test. I like the anonymity of the home test. (Sebastian)

You can hide this and, yes, an HIV test is the sort of thing you want to hide. It's safer. (Joe) 
The home test means I do it and nobody and I mean literally nobody knows the result because the test is discreet. (Peter)

Given the perceived stigma surrounding HIV testing, individuals generally sought to conceal their own experience of testing from other people. They did not wish to be seen in clinical or community settings where HIV testing was offered due to the fear that others might suspect that they had taken sexual risks or that they were HIV-positive. Accordingly, some men wished to avoid interpersonal interactions in relation to their sexual behavior or possible HIV status. HIV stigma plays a role in determining attitudes towards testing. Conversely, individuals who had taken advantage of self-testing generally evaluated this positively. As Sebastian and Joe indicated, self-testing enabled them to conceal their experience of testing from others. Peter noted that, while in clinical and community settings at least one other person (that is, the HIV tester) would know his HIV status, the home test enabled him to keep his HIV status entirely private. This served as a source of consolation for individuals who felt uneasy about disclosing their HIV status to others, including health professionals.

Some interviewees lamented the 'loss of control' associated with being tested for HIV in that they were reliant on the tester to reveal the outcome of the test. Conversely, self-testing was perceived to restore feelings of control:

It sounds silly but you sort of like lose control when you're waiting for your test. You're waiting for them to come up and tell you 'we have your results now' and you're sort of reliant on them having it. This is hard to deal with sometimes but with the home test you're not really at their mercy. Yes, you buy it but you've got it in your hands and you can look at the result whenever you want to. (Mark)

Feelings of self-efficacy could be challenged by the experience of testing in traditional settings. Interviewees described the anxiety-inducing experience of waiting for their test result in the knowledge that they had no control over the timing of the delivery of the result. One interviewee described his experience of taking an HIV test but then changing his mind because he felt 'not quite ready' to know the result. Mark described the loss of control associated with being tested for HIV as 'hard to deal with'. Conversely, self-testing at home appeared to restore his sense of self-efficacy in that he perceived greater feelings of control and agency over his HIV test and the timing of obtaining the result. The self-test enabled 
individuals to ascertain their HIV status at a time of their own choosing, that is, when they felt psychologically and emotionally 'ready' to know the result.

Like the other HIV testing contexts described in this article, the HIV self-test did also pose some psychosocial challenges. Fear of testing positive alone, devoid of social support, was a key concern:

For me, basically it's the fear that's always like got to me about going in for a test. There's the same fear when you have the home test. I've had it and before I did it thinking 'if the result is positive, who is going to tell me everything will be OK?' I was making scenarios in my head, all the usual doom and gloom basically. (Salim)

While interviewees highlighted the convenience of self-testing, like Salim they worried about the psychosocial consequences of testing positive for HIV alone at home. Salim indicated his need for reassurance about the prognosis of an HIV patient in case he did test positive. Interviewees did not appear to be psychologically prepared for a positive test result. This rendered the prospect of testing positive at home, alone, psychologically distressing. Conversely, a small number of interviewees identified the availability of social support (from qualified healthcare professionals) as an advantage of testing in the GUM clinic.

Participants expressed concerns about their ability to perform the test correctly and the possibility of receiving a false result:

I know the test is pretty easy to do but I'm sure I'd mess it up anyhow. I'm no good at this sort of stuff. So there's always that worry. What if it's a - what do you call it? false positive or something like that? I'd die. I'd just drop dead. (Jamie)

The fear of generating a false positive HIV test result due to erroneous use of the self-test led to trepidation about this method. Some therefore preferred to be tested in a 'reliable' context, e.g. GUM or community settings, notwithstanding the acknowledged limitations of testing in these contexts. Some interviewees also reported difficulties in believing their test result even in such 'reliable' contexts, which accentuated their concerns regarding the accuracy of self-testing kits: 
I'm just a bit $O C D$ and when I get the result from them [the health advisor] I'm like 'did you get my number down OK?' or maybe they messed up the test and I've really got it [HIV]... I'd probably not trust the result [of a self-test]. (Sam)

Given fears of testing positive for HIV, there was a desire for certainty about one's result, be it negative or positive.

\section{DISCUSSION}

The results of this qualitative study of 18 MSM's perceptions of HIV testing in 3 contexts provide nuanced insight into attitudes towards, and perceived facilitators and inhibitors of, HIV testing in these contexts. The decision about the context in which to test for HIV is likely to be moderated by a multitude of social and psychological factors. This study set out to explore some of these factors. The availability of HIV testing in a variety of contexts is likely to facilitate greater access to testing among individuals at risk of HIV.

Although most interviewees reported testing for HIV in GUM clinics, they identified a series of potential barriers to testing in this context. The perceived 'clinical atmosphere' of GUM clinics appears to activate the 'feared self' of illness in some individuals, which is at odds with the 'desired self' of wellness. This is consistent with the Possible Selves Theory (Markus \& Nurius, 1986), which differentiates between 'real' and hypothetical facets of identity, including one's actual, desired, and feared selves. Despite awareness of objective risk, individuals hope for and, in many cases, anticipate a negative test result. Accordingly, it is easy to see why HIV testing constitutes an anxiety-inducing experience for MSM (Lorenc et al., 2011). The anxiety surrounding HIV testing may be further accentuated by growing resource limitations in GUM clinics in the UK, resulting in delays in being seen by a healthcare professional. Following a trigger for HIV testing, such as a risk event, individuals will plausibly be in a heightened state of anxiety and delays may aggravate psychological distress and decrease willingness to test in the future.

Furthermore, in view of the stigma appended to sexual orientation and HIV, some MSM may express concern about involuntary disclosure of these aspects of identity if they are seen in GUM clinics or indeed community settings. The existence of social stressors, such as homonegativity, may lead some MSM to become aware of, and sensitive to, social stigma associated with their sexual orientation. Interviewees described experiences of perceived stigma from healthcare professionals in GUM clinics, which could lead to non-disclosure of key information regarding sexual behavior and, in some cases, to disengagement from 
sexual health services (Stutterheim et al., 2014). In previous research, it has been shown that MSM may resist the category 'high risk' because of the negative outcomes of this category for identity. In particular, this can induce feelings of low self-esteem and disrupt one's sense of continuity, especially if one has consistently viewed oneself as 'low risk' (Jaspal \& Daramilas, 2016). In order to maintain the identity of 'low risk', the individual may avoid seeking an HIV test in GUM clinics, which, for some MSM, evokes an illness identity. Identity process theory (Jaspal \& Breakwell, 2014) from social psychology predicts that individuals will seek to protect valued principles of identity, such as self-esteem, which can include disengagement from services.

Many of the concerns, which interviewees expressed in relation to testing in GUM clinics, were alleviated by testing in community settings. Individuals reportedly capitalized on the opportunity to test for HIV in community settings, which they used for other primary purposes. Given the shared identity aspects with HIV testers in community settings (e.g. a shared sexual identity), some MSM derived a sense of rapport with the testers and perceived no stigma from them. They generally felt more comfortable disclosing aspects of their sexual identity and behavior. Pre-test questioning was perceived to be less intrusive than that in GUM clinics. This may lead to accurate self-disclosure, greater readiness to derive social support prior to the HIV test, and more opportunities to discuss sexual identity, risk and wellbeing. Yet, there are also barriers to accessing testing in community settings. Some MSM may avoid LGBT community settings because they do not self-identify as LGBT or because they fear homophobic stigma if they are seen entering the premises. Furthermore, in this context too, MSM may resist the category of 'high risk' and wish to prevent others from making assumptions regarding their 'risky' sexual behavior.

Many of these barriers to testing appear to be interpersonal in origin. Individuals are concerned about what others will potentially think about them and their sexual behavior. HIV self-testing can obviate many of the barriers rooted in interpersonal relations given that it is performed, and the result is obtained, individually. Interviewees generally perceived self-testing to be a safe, discreet and confidential means of ascertaining one's HIV status with no risk of social stigma from others on the basis of sexual orientation or HIV positive status. However, the desire to limit interpersonal contact in the context of ascertaining one's HIV status may deprive the individual of social support in the event of a reactive HIV test result. Identity process theory (Jaspal \& Breakwell, 2014) describes the derivation of social support as an especially effective strategy for coping with psychological adversity, which is often the outcome of a positive HIV test result. Thus, individuals at risk of HIV must 
be supported prior to taking a test in order to enable them to consider what a reactive/ positive test result might mean for their identity, health and wellbeing. Furthermore, in the event of a negative test result, the individual ought to receive information about how best to maintain a negative serostatus.

\section{Recommendations}

On the basis of this qualitative study, it is possible to offer some recommendations for removing barriers and facilitating access to HIV testing among MSM.

- GUM clinics remain an important context in which MSM test for HIV. Individuals may perceive this context to be more accessible if it appears to be less clinical, as this might be less evocative of an illness identity and, thus, become associated with routine health screening/ HIV testing.

- Cuts to funding for sexual health services in the UK are likely to decrease staffing resources, leading to delays in service provision. This can accentuate the anxiety that tends to accompany HIV testing and lead to disengagement.

- HIV testing campaigns should continue to decouple the category 'high risk' from HIV testing, as this association may cause those individuals who do not, or who do not wish to, view themselves as high risk to disengage from HIV testing.

- Social support (e.g. counseling) is an important aspect of HIV testing. It must precede the HIV test in order to promote cognitions that accommodate a possible reactive test result and it must follow delivery of the result so that the patient can be empowered to cope effectively with the reactive result or to formulate behaviors that maintain a negative serostatus. This must be considered in the context of HIV testing in community settings and, especially, in the context of HIV self-testing where the individual performs the test and receives the result individually.

- The pre-test risk assessment and counseling offered to the individuals must be devoid of stigma. Healthcare practitioners should be mindful of the potential hypervigilance and hypersensitivity to stigma that MSM may manifest due to previous experiences of stigma. A positive HIV testing experience is likely to lead to consistent testing behavior.

- Continued efforts to de-stigmatize LGBT identities are likely to benefit efforts to promote HIV testing in community settings, which some MSM may avoid due to fears to homophobic stigma. 
- Approved HIV self-testing kits in the UK have a high degree of accuracy. The public should be informed about the high degree of accuracy to restore confidence in this approach to testing.

\section{Limitations}

Future research should explore attitudes towards distinct HIV testing venues using other methodological approaches, such as a quantitative survey design with a representative sample of MSM. This would enable us to generalize our findings which are currently applicable only to the current study sample. This study focused on MSM in London and the East Midlands of England, so future research ought to complement this study with data from other parts of the UK. Although we now have considerable knowledge about the barriers to HIV testing (as a practice), which is summarized in the introduction of this article, it is possible that there are barriers specific to MSM in the East Midlands. This should be investigated in future work. On a related note, all of the participants were recruited from sexual health charities and were thus engaged with sexual health issues. Future research should also attempt to capture the perspectives of those who do not engage with sexual health services. This study compares three distinct types of HIV testing venues. There is much diversity within them. Future research ought to explore each venue type in more depth, exploring attitudes towards testing in gay saunas vis-à-vis LGBT community centers, for instance. Furthermore, it must be acknowledged that not all of the participants had firsthand experience of testing in all of these contexts, which meant that some of their remarks were speculative. Future research should also focus on the attitudes of those with first-hand experience of these testing contexts.

\section{Conclusions}

HIV testing is the first step to promoting positive health outcomes in people living with HIV and to preventing onward HIV transmission. Although rates of testing in MSM are increasing, almost one tenth of HIV-infected individuals remain undiagnosed. HIV testing must continue to be offered in a wide range of contexts. GUM clinics, community settings and self-testing are key contexts for HIV testing. Greater access to HIV testing makes it more likely that people will test. Inevitably, some testing contexts will be preferable to some and undesirable to others. In this article, a series of evidence-based recommendations are offered in order to identify ways of rendering these testing contexts more desirable to MSM at risk of HIV. Yet, there will be many other contexts in which HIV testing is, and ought to be, 
offered. In the existing and emerging HIV testing contexts, we must continue review, identify and attempt to eradicate barriers, in order to ensure that MSM continue to test for HIV, to link patients to relevant services, and, crucially, to realize our ambition of halting the transmission of HIV.

\section{REFERENCES}

Bingham, T., Secura, G., King, C., Lozano, A., Simon, P., \& Bunch, G. (2003). HIV prevalence and risk behaviors among men seeking HIV testing and prevention services inside Los Angeles bathhouses. Paper presented at: National HIV Prevention Conference, 27-30 July 2003, Atlanta, G.A. Abstract T2-B0504.

Bond, K.T., Frye, V., Taylor, R., Williams, K., Bonner, S., Lucy, D., et al., for the Straight Talk Study Team. (2015). Knowing is not enough: A qualitative report on HIV testing among heterosexual African-American men. AIDS Care, 27(2), 182-188.

Edwards, N., Peterson, W.E. \& Davies, B.L. (2006). Evaluation of a multiple component intervention to support the implementation of a 'Therapeutic Relationships' best practice guideline on nurses' communication skills. Patient Education \& Counseling, 63, 3-11.

Evangeli, M., Pady, K. \& Wroe, A.L. (2016). Which psychological factors are related to HIV testing? A quantitative systematic review of global studies. AIDS \& Behavior, 20, 880-918.

Figueroa, C., Johnson, C., Verster, A. \& Baggaley, R. (2015). Attitudes and acceptability on HIV self-testing among key populations: A literature review. AIDS \& Behavior, 19, 1949-65.

Goodall, L. \& Leen, C. (2011). Late diagnosis of HIV: Could this be avoided? Scottish Medical Journal, 56(2), 84-86.

Gray, R.T., Prestage, G.P., Down, I., Ghaus, M.H., Hoare, A., Bradley, J. \& Wilson, D.P. (2013). Increased HIV testing will modestly reduce HIV incidence among gay men in NSW and would be acceptable if HIV testing becomes convenient. PLOS ONE, 8(2), e55449.

DOI:10.1371/journal.pone.0055449.s010

Heijnders, M. \& Van Der Meij, S. (2006). The fight against stigma: An overview of stigmareduction strategies and interventions. Psychology, Health \& Medicine, 11, 353-363.

Hsieh-Fang, H. \& Shannon, S.E. (2005). Three approaches to qualitative content analysis. Qualitative Health Research, 15(9), 1277-88.

Jaspal, R. \& Daramilas, C. (2016). Perceptions of pre-exposure prophylaxis (PrEP) among HIVnegative and HIV-positive men who have sex with men. Cogent Medicine, 3, 1256850.

DOI:10.1080/2331205X.2016.1256850

Jaspal, R. (2018). Enhancing Sexual Health, Self-Identity \& Wellbeing among Men Who Have Sex With Men: A Guide for Practitioners. London: Jessica Kingsley Publishers. 
Johnson, C., Baggaley, R., Forsythe, S., van Rooyen, H., Ford, N., Mavedzenge, S.N., Corbett, E., Natarajan, P. \& Taegtmeyer, M. (2014). Realizing the potential for HIV self-testing. AIDS \& Behavior, 18, S391-S395

Kall, M. M., Smith, R. D., \& Delpech, V. C. (2012). Late HIV diagnosis in Europe: A call for increased testing and awareness among general practitioners. European Journal of General Practice, 18, 181-186.

Kendrick, S.R., Kroc, K.A., Withum, D., Rydman, R.J., Branson, B. \& Weinstein, R.A. (2005). Outcomes of offering rapid point-of-care HIV testing in a sexually transmitted disease clinic. JAIDS, 38, 142-6.

Kirwan, P.D., Chau, C., Brown, A.E., Gill, O.N., Delpech, V.C. and contributors (2016). HIV in the UK - 2016 report. December 2016. Public Health England, London.

https://www.gov.uk/government/uploads/system/uploads/attachment data/file/602942/H IV in the UK report.pdf Accessed 2 December 2017.

Kurka, T., Soni, S. \& Richardson D. (2015). P107 Sexual health services for men who have sex with men (MSM): are they acceptable? Sexually Transmitted Infections, 91, A51.

Li, X., Lu, H., Ma, X., Sun, Y., He, X., Li, C. \& Jia Y. (2012). HIV/AIDS-related stigmatizing and discriminatory attitudes and recent HIV testing among men who have sex with men in Beijing. AIDS \& Behavior, 16, 499-507.

Lorenc, T., Marrero-Guillamón, I., Llewellyn, A., Aggleton, P., Cooper, C., Lehmann, A. \& Catriona, L. (2011). HIV testing among men who have sex with men (MSM): Systematic review of qualitative evidence, Health Education Research, 26(5), 834-846.

MacKellar, D.A., Valleroy, L.A., Secura, G.M., Behel, S., Bingham, T., Celentano, D.D., Koblin, B.A., Lalota, M., McFarland, W., Shehan, D., Thiede, H., Torian, L.V., Janssen, R.S. and Young Men's Survey Study Group. (2005). Unrecognized HIV infection, risk behaviors, and perceptions of risk among young men who have sex with men: opportunities for advancing HIV prevention in the third decade of HIV/AIDS. Journal of Acquired Immune Deficiency Syndromes, 15(38), 603-14.

Marcus, U., Gassowski, M., \& Drewes, J. (2016). HIV risk perception and testing behaviours among men having sex with men (MSM) reporting potential transmission risks in the previous 12 months from a large online sample of MSM living in Germany. BMC Public Health, 16, 1111. DOI:10.1186/s12889-016-3759-5

McDaid, L.M. Aghaizu, A., Frankis, J., Riddell, J. Nardone, A., Mercey, D., Johnson, A.M., Hart, G.J. \& Flowers, P. (2016) Frequency of HIV testing among gay and bisexual men in the UK: Implications for HIV prevention. HIV Medicine, 17, 683-693.

Newcomb, M.E. \& Mustanski, B. (2010). Internalized homophobia and internalizing mental health problems:isepala meta-analytic review. Clinical Psychology Review, 30(8), 1019-1029.

Nuwaha, F., Kabatesi, D., Muganwa, M. \& Whalen, C.C. (2002). Factors influencing acceptability of voluntary counselling and testing for HIV in Bushenyi district of Uganda. East African Medical Journal, 79, 626-632. 
Prost, A., Chopin, M., McOwan, A., Elam, G., Dodds, J., Macdonald, N. \& Imrie, J. (2007).

"There is such a thing as asking for trouble": Taking rapid HIV testing to gay venues is fraught with challenges. Sexually Transmitted Infections, 83(3), 185-188.

Rüsch, N., Corrigan, P.W., Wassel, A., Michaels, P., Larson, J.E., Olschewski, M., Wilkniss, S. \& Batia, K. (2009) Self-stigma, group identification, perceived legitimacy of discrimination and mental health service use. British Journal of Psychiatry, 195, 551-552.

St Lawrence, J.S., Kelly, J.A., Dickson-Gomez, J., Owczarzak, J., Amirkhanian, Y.A. \& Sitzler, C. (2015). Attitudes toward HIV voluntary counseling and testing (VCT) among African American men who have sex with men: Concerns underlying reluctance to test. AIDS Education \& Prevention, 27(3), 195-211

Spielberg, F., Branson, B.M., Goldbaum, G.M., Kurth, A. \& Woods, R.W. (2003). Designing an HIV counseling and testing program for bathhouses: The Seattle experience with strategies to improve acceptability. Journal of Homosexuality, 44(3-4), 203-20.

Stutterheim, S.E., Sicking, L., Brands, R., Baas, I., Roberts, H., van Brakel, W.H., Lechner, L., Kok, G. \& Bos, A.E.R. (2014). Patient and provider perspectives on HIV and HIV-related stigma in Dutch health care settings. AIDS Patient Care and STDs, 28(12), 652-665.

The INSIGHT START Study Group (2015) Initiation of antiretroviral therapy in early asymptomatic HIV infection. New England Journal of Medicine, 373, 795-807.

Thornton, A.C., Delpech, V., Kall, M.M. \& Nardone, A. (2012). HIV testing in community settings in resource-rich countries: a systematic review of the evidence. HIV Medicine, 13(7), 416-26.

Witzel, T.C., Melendez-Torres, G.J., Hickson, F., et al (2016a). HIV testing history and preferences for future tests among gay men, bisexual men and other MSM in England: results from a cross-sectional study. BMJ Open, 6, e011372. DOI:10.1136/bmjopen-2016011372

Witzel, T.C., Rodger, A.J., Burns, F.M., Rhodes, T. \& Weatherburn, P. (2016b). HIV self-testing among men who have sex with men (MSM) in the UK: A qualitative study of barriers and facilitators, intervention preferences and perceived impacts. PLOS ONE, 11(9), e0162713. DOI:10.1371/journal.pone.0162713

Wong, L.P. (2013) Multi-ethnic perspective of uptake of HIV testing and HIV-related stigma: A cross-sectional population-based study. AIDS Care, 25, 1356-1369.

Woods, W.J., Sabatino, J., Bauer, P.L., Adler, B., Dilley, J.W. \& Binson, D. (2000). HIV testing in gay sex clubs. International Journal of STDs \& AIDS, 11, 173-175.

Young, S.D., Nussbaum, A.D. \& Monin, B. (2007). Potential moral stigma and reactions to sexually transmitted diseases: evidence for a disjunction fallacy. Personality \& Social Psychology Bulletin, 33, 789-799. 